\title{
Layered shielding design for an active neutron interrogation system
}

Zachary D. Whetstone, Kimberlee J. Kearfott (corresponding author: kearfott@umich.edu)

Department of Nuclear Engineering and Radiological Sciences, University of Michigan, 2355

Bonisteel Boulevard, 1943 Cooley Building, 48109-2104 Ann Arbor, MI USA

\begin{abstract}
The use of source and detector shields in active neutron interrogation can improve detector signal. In simulations, a shielded detector with a source rotated $\pi / 3$ radians relative to the opening decreased neutron flux roughly three orders of magnitude. Several realistic source and detector shield configurations were simulated. A layered design reduced neutron and secondary photon flux in the detector by approximately one order of magnitude for a deuterium-tritium source. The shield arrangement can be adapted for a portable, modular design.
\end{abstract}

\section{Keywords}

Active neutron interrogation; radiation shielding; neutron detection; gamma ray detection; portable shielding; shielding simulations 


\section{Introduction}

There have been several active neutron interrogation methods proposed to detect explosives and narcotics (Cinausero et al., 2004; Clifford et al., 1999; Lehnert and Kearfott, 2010; Whetstone and Kearfott; 2014). The analyzed signal consists of one or more emitted radiation types, including characteristic x-rays, gamma rays, and inelastic and elastically scattered neutrons. Most neutron sources employed are approximately isotropic, which is undesirable because of the increased production of secondary radiation that adds noise. Methods may involve neutrons sources with a variety of energies, with recent research showing the potential for tuned neutron generator beams (Whetstone, 2015; Whetstone and Kearfott, 2015).

By designing the shield for an isotropic source with a single opening, a limited neutron beam can be created. The neutrons emitted towards the opening have an unattenuated path, while many neutrons emitted at other angles are scattered and absorbed in the shielding (Whetstone and Kearfott, 2011). Similarly, the detectors used in an active interrogation system can be shielded so that radiation from the area of interest will be preferentially detected. Using both source and detector shields creates a cleaner signal for both photons and neutrons, improving determination of the target's composition for a variety of methods. Such improved interrogating source beams may be particularly useful for newly proposed methods that do not necessarily involve time-of-flight analysis, and rely upon simple flags based upon measurements of a variety of different analyzed signals (Lehnert et al., 2012, Lehnert and Kearfott, 2011b; Lehnert and Kearfott, 2014, Lehnert and Kearfott, 2015; Valkovic et al., 2015; Whetstone and Kearfott, 2011).

The shield designs employed in this work were similar to those presented previously for shielding a deuterium-tritium (D-T) source and based upon much earlier work (Green and Thomas, 1969; Maruyama and Bouts, 1972; Whetstone and Kearfott, 2011). After evaluating several designs, two were chosen for further exploration. The neutron source shields were $0.5 \mathrm{~m}$ thick and consisted either of polyethylene or alternating layers of steel and polyethylene. The polyethylene is a moderator that slows neutrons, increasing the likelihood of absorption. Steel scatters and absorbs the neutrons while also shielding secondary gamma rays created within the environment or shield.

For neutrons in the multiple $\mathrm{MeV}$ range, the iron atoms in steel have larger scatter cross sections than low atomic number materials (Shultis and Faw, 1996). The energy lost by the 
neutron in an elastic scatter with iron is minimal, but by having multiple layers of the low and middle atomic number materials, the neutrons will have many chances to scatter and pass through moderating material to lose energy or be absorbed. The iron also has a large (n, $\gamma$ ) cross section near $1.15 \mathrm{keV}$, resulting in significant neutron absorption.

The same layered technique used to shield the source neutrons can be applied to detectors, only in a reverse order. The processes involved in attenuating the neutrons emerging from the source will also work on neutrons directed toward a detector. In this case, since the neutrons are approaching from the outside, a reversal of the source shield arrangement was chosen. Any neutrons that are not coming from the target area will have to pass through shielding, where they can be scattered, slowed, or absorbed by the polyethylene and steel, reducing the probability of entering the detector. The steel will also shield the detector from gamma rays. The result is a detector with a limited field of view focused on the area of interest.

The purpose of this work was to extend the layered steel and polyethylene design to use with a detector in an active neutron interrogation system. An examination was also conducted on how the combined source and detector shields affected the neutrons and photons entering the detector. Additional discussion on modular implementation of the design is included.

\section{Experimental}

Monte Carlo N-Particle 5 (MCNP 5) ${ }^{1}$ was used to perform multiple simulations to determine neutron transmission. The shielded detector was suspended in air with an isotropic neutron source placed $1.0 \mathrm{~m}$ from the shield center and rotated $0, \pi / 6, \pi / 3$, and $\pi / 2$ radians about the opening. The neutron energies simulated were 14.1, 12.0, 10.0, 8.0, 6.0, 4.0, 2.5, and 1.0 $\mathrm{MeV}$. For each energy and source position, an F4 tally, representing the flux averaged over the cell, was estimated for neutrons in the detector volume. The number of histories performed ensured $<5 \%$ statistical uncertainty.

A shield previously developed for a D-T neutron source (Whetstone and Kearfott, 2011) was adapted for use with the detector by reversing the layering. The layer of shield closest to the detector was $0.05 \mathrm{~m}$ polyethylene, followed by $0.05 \mathrm{~m}$ steel, $0.05 \mathrm{~m}$ polyethylene, $0.05 \mathrm{~m}$ steel, $0.05 \mathrm{~m}$ polyethylene, $0.20 \mathrm{~m}$ steel, and, finally, $0.05 \mathrm{~m}$ polyethylene on the outside. The detector was modeled after a $0.127 \mathrm{~m}$ radius, $0.127 \mathrm{~m}$ long liquid scintillator, EJ-309, commonly used for fast neutron measurements (Kaplan et al., 2013; Dolan, et al., 2014). The $0.50 \mathrm{~m}$ thick, 0.127 inner radius, $0.50 \mathrm{~m}$ long shield, shown in Fig. 1, is open at one end to allow unattenuated 
radiation to approach the detector. Material compositions are in Table 1.

Table 1 Description of materials simulated, with selected data from the literature [17-18]

\begin{tabular}{|c|c|c|c|}
\hline Material & $\begin{array}{l}\text { Elemental makeup (relative } \\
\text { abundance) }\end{array}$ & Elemental makeup (weight \%) & $\begin{array}{l}\text { Density } \\
\left(\mathrm{kg} \mathrm{m}^{-3}\right)\end{array}$ \\
\hline $\begin{array}{l}\text { Air (McConn et } \\
\text { al., 2011) }\end{array}$ & & $\begin{array}{l}\mathrm{C}(0.001), \mathrm{N}(0.755), \mathrm{O}(0.231) \\
\operatorname{Ar}(0.013)\end{array}$ & 1.2 \\
\hline Polyethylene & $\mathrm{H}(4), \mathrm{C}(2)$ & & 940 \\
\hline Water & $\mathrm{H}(2), \mathrm{O}(1)$ & & 1,000 \\
\hline Steel & & $\mathrm{C}(0.2), \mathrm{Fe}(99.8)$ & 7,860 \\
\hline EJ-309 1 & $\mathrm{H}(5), \mathrm{C}(4)$ & & 960 \\
\hline Nitrogen & $\mathrm{N}(1)$ & & 810 \\
\hline $\begin{array}{l}\text { Soil (Eckerman } \\
\text { and Ryman, } \\
\text { 1993) }\end{array}$ & $\begin{array}{l}\mathrm{H}(0.2938), \mathrm{C}(0.0187), \mathrm{O} \\
(0.5045), \mathrm{Al}(0.0259), \mathrm{Si} \\
(0.1354), \mathrm{K}(0.0143), \mathrm{Fe} \\
(0.0027)\end{array}$ & & 1,750 \\
\hline $\begin{array}{l}\text { Concrete } \\
\text { (McConn et al., } \\
\text { 2011) }\end{array}$ & & $\begin{array}{l}\mathrm{H}(0.56), \mathrm{O}(49.81), \mathrm{Na}(1.71), \\
\mathrm{Mg}(0.26), \mathrm{Al}(4.57), \mathrm{Si}(31.51) \\
\mathrm{S}(0.13), \mathrm{K}(1.92), \mathrm{Ca}(8.29), \mathrm{Fe} \\
(1.24)\end{array}$ & 2,080 \\
\hline
\end{tabular}

The combined source and detector shields were also simulated in a generic, more realistic environment that included an interrogated object, shown in Fig. 2. The $0.634 \mathrm{~m}$ radius source shield is either the layered design studied previously or strictly polyethylene (Whetstone and Kearfott, 2011). The shield barrel has a radius of $0.134 \mathrm{~m}$ instead of $0.160 \mathrm{~m}$ presented previously, providing a $\pi / 6$ steradian solid angle from the source to the inner shield edge. To approximate a neutron transmission measurement, the barrels of the two shields are parallel to the ground and facing each other with the source $1.873 \mathrm{~m}$ from the detector face. A $0.40 \mathrm{~m}$ radius, $0.30 \mathrm{~m}$ long water cylinder placed between the source and the detector represents an interrogation target. It is several mean free paths thick for D-T neutrons and wide enough that all 
unattenuated neutrons from the source shield opening will pass through. The source and center of the detector are $0.85 \mathrm{~m}$ above the $0.15 \mathrm{~m}$ thick concrete floor resting on soil. The walls and ceiling of the $5.50 \times 6.00 \mathrm{~m}, 2.35 \mathrm{~m}$ tall room are $0.15 \mathrm{~m}$ thick concrete. Several simulations were run with various shield configurations named arrangements 1-6, and one configuration with no shields, referred to as air. The specific layering schemes can be seen in Fig. 3. Only $14.1 \mathrm{MeV}$ D-T neutrons were studied: the shield design could be adapted for the less energetic deuteriumdeuterium neutrons. An F4 tally determined the energy-dependent neutron flux averaged over the detector volume. Another F4 tally evaluated the effectiveness of the various shielding arrangements to minimize the secondary gamma ray background. The number of histories simulated for each arrangement varied so the statistical uncertainty of each energy bin was below $10 \%$. Generally it was significantly lower.

The neutron flux for the realistic arrangement was also investigated with the source and detector offset $\pi / 2$ radians in the horizontal plane, meeting the requirements of Neutron Elastic Scatter or Pulsed Fast Neutron Analysis measurements (Lehnert and Kearfott, 2011a). In this case, source neutrons that enter the target must interact at least once before they are directed towards the detector. They can also interact in the environment, or pass through both source and detector shielding before entering the detector. The neutron target was changed from a disc of water to a $0.25 \mathrm{~m}$ radius sphere of liquid nitrogen to yield a more obvious scattered neutron spectrum. Relative to Fig. 2, the source and its shield were rotated about the center of the target $\pi / 2$ radians counter clockwise and moved back an additional $0.15 \mathrm{~m}$ to allow space for both the source and detector shields. The source was $1.15 \mathrm{~m}$ from the target center. The detector/shield locations and dimensions remained unchanged. Each arrangement was again simulated, with the source and detector shield from arrangement 1 now referred to as arrangement 7, arrangement 2 as 8, etc. A modified arrangement 5, with the shield openings aligned, was chosen to compare to the rotated simulations. Arrangement 5 was considered a compromise, using polyethylene in the source shield to minimize mass and reduce the generation of secondary gamma rays, while the detector shield still employed layered polyethylene and steel to shield both neutrons and gamma rays. The only modification to arrangement 5 was to move the source and shield back $0.15 \mathrm{~m}$ and change the scatter target to match arrangements 7-12.

\section{Results}

The averaged detector neutron flux from the simulated sources at various energies and 
angles and without a target present can be found in Fig. 4. The largest flux was at 0 radians relative to the shield opening and the smallest was at $\pi / 3$ radians. The flux difference between the two was estimated to be roughly three orders of magnitude for all neutron energies, both with the steel and polyethylene layered shield and the shield that contained only polyethylene.

The results for the aligned system simulated in a room can be seen in Fig. 5 and 6. The histograms were normalized by the width of each bin and shown as scatter plots, where a data point represents the upper right corner of each bin. The spectra from D-T neutrons entering the detector were very similar, regardless of the detector and source shield. Only the simulation with no shield, labeled air, showed a flux spectrum approximately one order of magnitude greater for all energies. For the shielded arrangements, the ratio of counts in the peak to the counts for energy bins between 4 and $12 \mathrm{MeV}$ were all similar and ranged from 21 to 36. The air arrangement, with no shield material, had ratios between 7 and 19.

The secondary photon spectra showed a greater variability with shield arrangement. Again, the largest overall flux was the simulation without shielding, while the lowest flux was seen for arrangements 3 and 5 at higher energies, and arrangement 2 for lower energies. The flux difference between shielded configurations was just over an order of magnitude between 6 and 7 $\mathrm{MeV}$ and got progressively smaller at lower energies. The photon spectra were shown for energies up to $7 \mathrm{MeV}$ to account for the most prominent gamma rays associated with inelastic neutron scatter off carbon in the shielding material and oxygen in the interrogation target. In addition, there are gamma rays resulting from thermal neutron absorption in hydrogen found in both the target and shield. The gamma rays of concern are hydrogen: $2.22 \mathrm{MeV}$, carbon: 4.43 $\mathrm{MeV}$, and oxygen: $6.13 \mathrm{MeV}$, and are all seen in the simulations. An estimate of the peak to background ratio at all three energies was performed to better understand the characteristic gamma ray behavior of the various arrangements, with results in Table 2.

Table 2 Peak to background ratio for characteristic gamma rays in simulated D-T shielding arrangements with the source and detector shield openings aligned

\begin{tabular}{lccccccc}
\hline $\begin{array}{l}\text { Photon } \\
\text { energy } \\
(\mathrm{MeV})\end{array}$ & 1 & 2 & 3 & 4 & 5 & 6 & Air \\
\cline { 2 - 8 } & \multicolumn{6}{c}{ Peak to background ratio for each arrangement } \\
\hline 2.31 & $9.2 \pm 0.2$ & $17.3 \pm 0.4$ & $14.2 \pm 0.2$ & $12.0 \pm 0.1$ & $12.8 \pm 0.3$ & $10.7 \pm 0.3$ & $7.0 \pm 0.1$
\end{tabular}




\begin{tabular}{rrrrrrrr}
4.43 & $9.4 \pm 0.3$ & $9.5 \pm 0.4$ & $39.9 \pm 1.7$ & $7.1 \pm 0.2$ & $29.9 \pm 1.5$ & $11.1 \pm 0.5$ & $3.6 \pm 0.1$ \\
6.13 & $3.3 \pm 0.1$ & $7.9 \pm 0.5$ & $22.3 \pm 1.6$ & $10.1 \pm 0.4$ & $9.2 \pm 0.6$ & $3.7 \pm 0.2$ & $9.5 \pm 0.2$ \\
\hline
\end{tabular}

When rotated $\pi / 2$ radians, compared to the previous simulations, a large decrease in the 14.1 MeV neutrons was observed in arrangements $7-12$. The simulated detector neutron flux of arrangements 7 - 12 was normalized by the flux of the modified arrangement 5 and displayed in Fig. 7. For arrangements 7, 9, 11, and 12, which all included source shields, the ratios at 14.1 $\mathrm{MeV}$ are below 0.001 . The ratios for arrangements 8 and 10, which had no source shields, were below 0.06 . Large peaks at $12.6 \mathrm{MeV}$, with magnitudes between 1.2 and 0.7 are visible in all spectra.

The gamma ray spectra for the various rotated arrangements are not shown because the magnitude and shape were similar to the aligned spectra. However, an estimate of the peak to background ratio was again performed, with results in Table 3.

Table 3 Peak to background ratio for characteristic gamma rays in simulated D-T shielding arrangements with the shielded detector rotated $\pi / 2$ radians relative to the source

\begin{tabular}{|c|c|c|c|c|c|c|c|c|}
\hline \multirow{2}{*}{$\begin{array}{l}\text { Photon } \\
\text { energy } \\
(\mathrm{MeV})\end{array}$} & \multicolumn{8}{|c|}{ Peak to background ratio for each arrangement } \\
\hline & 7 & 8 & 9 & 10 & 11 & 12 & Air & Modified 5 \\
\hline 2.31 & $24.6 \pm 0.6$ & $13.4+0.2$ & $13.6 \pm 0.3$ & $13.3+0.2$ & $19.1 \pm 0.5$ & $18.8+0.4$ & $6.1 \pm 0.1$ & $13.9 \pm 0.2$ \\
\hline 4.43 & $4.9 \pm 0.2$ & $5.1+0.1$ & $23.5 \pm 1.5$ & $13.7 \pm 0.4$ & $6.9 \pm 0.3$ & $6.5 \pm 0.3$ & $12.4 \pm 0.4$ & $21.6 \pm 0.6$ \\
\hline 6.13 & $1.8 \pm 0.1$ & $2.0 \pm 0.1$ & $3.2 \pm 0.4$ & $6.6 \pm 0.3$ & $1.8 \pm 0.2$ & $1.7 \pm 0.1$ & $8.5 \pm 0.4$ & $1.8 \pm 0.1$ \\
\hline
\end{tabular}

\section{Discussion}

\subsection{Simulations}

As seen in Fig. 4, both shielding arrangements, as a function of position, produce spectra having the same order of magnitude and basic shape. Rotating away from the opening, the neutrons passed through more material up to $\pi / 3$ radians, then less at $\pi / 2$ radians. In general, this correlated to the relative counts at each position in both shields. The order of magnitude drop in neutron flux from 0 to $\pi / 6$ radians and even larger drop from $\pi / 6$ to $\pi / 3$ radians demonstrate efficient detector collimator design.

The simulated neutron energy spectra from neutron transmission, seen in Fig. 5, reveal no 
significant differences among designs. For all arrangements, there is a peak at 14.1, then the spectra drops by over an order of magnitude and rises up to $10^{8}$ times as the energy approaches thermal. Even in arrangements 2 and 4, having no source shielding, the spectra are similar. The air arrangement, however, was consistently at least an order of magnitude greater than the shielded simulations and had a smaller peak to background ratio, validating the effectiveness of the detector shields at minimizing multiply scattered neutrons.

When the source was rotated about the target $\pi / 2$ radians in the horizontal plane, there were some interesting results. In Fig. 7, when the energy dependent neutron fluxes of arrangements 7-12 were normalized by the flux of modified arrangement 5, a peak at $12.6 \mathrm{MeV}$ resulting from single elastic scatter in the nitrogen target was clearly visible. The peaks have a magnitude ranging from 1.2 to 0.75 . Arrangements $7,9,11$, and 12, which included source shields, had peak ratios near 0.75 with similar spectra shapes. At higher energies, the ratios fell below 0.001, a three order of magnitude drop in source neutrons entering the detector. Despite signal noise, the simulation demonstrates a clear method of neutron energy adjustment. All that is required is an elastic scatter target of known atomic mass, a neutron source with a constant energy, and proper shielding to limit the detector field of view. This would allow for active interrogation with neutrons of controllable and variable energies.

The photon spectra for both the aligned and rotated arrangements varied significantly. The smallest spectra with aligned shields (arrangements 2, 3, and 5) have no steel in the source shield, eliminating many of the photons generated. Arrangement 4 also has no steel. However, more secondary photons from neutron interactions in the room are evident due to decreased attenuation in the polyethylene detector shield. At lower energies, the number of gamma rays entering the detector is similar, regardless of the lack or presence of shielding materials. This is because some of the higher energy gamma rays from the surrounding environment will scatter in the shield and lose energy, but ultimately, still enter the detector. This increases the lower energy photon flux, counteracting the expected reduction due to the presence of a shield.

Furthermore, as seen in Tables 2 and 3, the characteristic carbon gamma ray peak to background ratio is greatest for arrangements 2, 5, and 9, where the large carbon content of the polyethylene shields creates a sizeable $4.43 \mathrm{MeV}$ gamma ray peak. For the water interrogation target, arrangement 3 had the largest peak to background ratio for characteristic oxygen gamma rays at $6.13 \mathrm{MeV}$. When the detector and shield were rotated $\pi / 2$ radians, the carbon and oxygen 
peak to background ratios were generally smaller because the gamma rays had to pass through more shielding material. Arrangement 7, which had the steel and polyethylene layered shield for both the source and detector, provided the greatest peak to background ratio for the $2.22 \mathrm{MeV}$ hydrogen thermal neutron absorption gamma rays. With the large amounts of steel present, the amount of hydrogen present was reduced while the arrangement effectively limited the detector field of view.

\subsection{Proposed shielding design}

The addition of a source and detector shield to an active neutron interrogation system helps minimize undesired neutrons and photons entering the detector. When the source and detector shield openings are facing each other, the relative arrangement of steel and polyethylene around the source does not have a significant effect on neutron flux in the detector. Although steel in the source shield reduced the neutron flux and total dose around an isotropic neutron source, its removal significantly decreased the total detected secondary gamma ray flux (Whetstone and Kearfott, 2011). However, a larger characteristic carbon gamma ray peak will be present, which could be a problem for some measurements. Similarly, the use of steel in the detector shield does not appear to meaningfully change the detector’s neutron spectra.

When the detector is rotated $\pi / 2$ radians, layers of steel around both the source and detector minimize environmental neutron interactions and secondary gamma rays entering the detector. This allows for cleaner signals from the target of interest. When used properly, steel can improve the performance of an active neutron interrogation system by effectively collimating the source and detector.

Appropriate collimation reduces the system footprint. The use of multiple separate layers allows for an easy method to break down and reconstruct the system, facilitating portability. This can be accomplished by using a rectilinear shield design, seen in Fig. 8. Each layer can consist of a preferred material in two "L" shaped pieces that fit together in an open-ended box. One end can be closed using a shielding cap, with the other left open. The next layer fits flush against the previous until the desired shield thickness is achieved. As seen in Fig. 9, the modular shield design can employ interlocking pieces, adding structural stability and eliminating the emergence of unattenuated neutrons at joints or between layers. The modular construction also allows for simple adjustment by changing, removing, or adding layers depending on the application.

\section{Conclusions}


The use of source and detector shielding can greatly increase the effectiveness of active neutron interrogation systems. The source shield limits unattenuated neutrons entering the surrounding area and, combined with the detector shield, reduces the number and energy of secondary photons and scattered neutrons entering the detector by an order of magnitude. The limited field of view provides a cleaner signal without sacrificing detector efficiency for the area of interest. The simulations show the similarities and differences between shield designs that employ either polyethylene or alternating layers of steel and polyethylene.

Depending on the application, the source and detector shields can be used separately or combined, with or without steel, to enhance an active neutron interrogation system. The proposed modular shield component designs allow for easy construction, transportation, disassembly and modification. The shield designs presented are viable options for active neutron interrogation and deserve consideration for new systems.

\section{Acknowledgements}

This research was performed under appointment to the Nuclear Nonproliferation International Safeguards Graduate Fellowship Program sponsored by the National Nuclear Security Administration's Next Generation Safeguards Initiative (NGSI).

This work was funded in-part by the Consortium for Verification Technology under the Department of Energy National Nuclear Security Administration, award number DENA0002534. 


\section{References}

Cinausero, M., Lunardon, M., Nebbia, G., Pesente, S., Viesti, G., Filippini, V., 2004.

Development of a thermal neutron sensor for humanitarian demining. Appl. Radiat. Isot. 61, 59-66. doi:10.1016/j.apradiso.2004.02.017

Clifford, E., Ing, H., McFee, J., Cousins, T., 1999. High rate counting electronics for a thermal neutron analysis land mine detector. Penetrating Radiation Systems and Applications 3769, 155-166. doi:10.1117/12.363677

Dolan. J.L., Marcath, M.J., Flaska, M., Pozzi, S.A., Chichester, D.L., Toanin, A., Peerani, P., 2014. Active-interrogation measurements of fast neutrons from induced fission in lowenriched uranium. Nucl. Instrum. Methods Phys. Res. Sect. A 738, 99-105. doi:10.1016/j.nima.2013.11.052

Eckerman, K.F., Ryman, J.C., 1993. External exposure to radionuclides in air, water, and soil. Oak Ridge National Laboratory, Report No. EPA-402-R-93-081.

Greene, D., Thomas, R.L., 1969. The attenuation of $14 \mathrm{MeV}$ neutrons in steel and polyethylene. Phys. Med. Biol. 14, 45-54. doi:10.1088/0031-9155/14/1/003

Kaplan, A.C., Flaska, M., Enqvist, A., Dolan, J.L., Pozzi, S.A., 2013. EJ-309 pulse shape discrimination performance with a high gamma-ray-to-neutron ratio and low threshold. Nucl. Instrum. Methods Phys. Res. Sect. A 729, 463-468. doi:10.1016/j.nima.2013.07.081

Lehnert, A.L., Kearfott, K.J., 2010. The detection of explosive materials: review of considerations and methods. Nucl. Technol. 172, 325-334.

Lehnert, A.L., Kearfott, K.J., 2011a. Simplified simulation of fast neutron scattering for an explosives detection application. Nucl. Sci. Eng. 168, 278-286. doi: 10.13182/NSE10-13

Lehnert, A.L., Kearfott, K.J., 2011b. Preliminary identification of flags for a novel algorithmbased approach for explosives detection using neutron interrogation for a simulated idealized cargo container scenario. Nucl. Instrum. Methods Phys. Res. Sect. A 638, 201-205. doi:10.1016/j.nima.2011.02.079

Lehnert, A.L., Flaska, M., Kearfott, K.J., 2012. DD neutron scatter measurements for a novel explosives detection technique. Nucl. Instrum. Methods Phys. Res. Sect. A 693, 195-202. doi:10.1016/j.nima.2012.07.047

Lehnert, A.L., Kearfott, K.J., 2014. Simulation for developing a flag-based active neutron interrogation method for explosives detection in sea land cargo containers. Nucl. Technol. 
188, 97-111. doi: 10.13182/NT11-125

Lehnert, A.L., Kearfott, K.J., 2015. A flag-based algorithm and associated neutron interrogation system for the detection of explosives in sea land cargo containers. Radiat. Phys. Chem. 112, 13-21. doi:10.1016/j.radphyschem.2015.02.026

Maruyama, T., Bouts, C.J., 1972. Attenuation of $15 \mathrm{MeV}$ neutrons in multilayer shields composed of steel, polyethylene and borated materials. Phys. Med. Biol. 17, 420-424. doi: 10.1088/0031-9155/17/3/010

McConn Jr, R.J., Gesh, C.J., Pagh, R.T., Rucker, R.A., Williams III, R.G., 2011. Compendium of material composition data for radiation transport modeling. Pacific Northwest National Laboratory, Report No. PNNL-15870.

Shultis, J.K., Faw, R.E., 1996. Radiation Shielding. Prentice Hall, Upper Saddle River, New Jersey.

Valkovic, V., Sudac, D., Nad, K., Obhodas, 2015. Container inspection in the port container terminal by using $14 \mathrm{MeV}$ neutrons. IEEE Tans. Nucl. Sci., In Press. doi: 10.1109/TNS.2016.2521925

Whetstone, Z.D., 2015. Designing and Implementing a Variable Energy Neutron System to Search for Conventional Explosives. Ph.D. Dissertation. Located: https://deepblue.lib.umich.edu/handle/2027.42/113596

Whetstone, Z.D., Kearfott, K.J., 2011. Use of multiple layers of repeating material to effectively collimate an isotropic neutron source. Nucl. Technol. 176, 395-413. doi: 10.13182/NT10-118

Whetstone, Z.D., Kearfott, K.J., 2014. A review of conventional explosives detection using active neutron interrogation. J. Radioanal. Nucl. Chem. 301, 629-639. doi:10.1007/s10967014-3260-5

Whetstone, Z.D., Kearfott, K.J., 2015. A method for using neutron elastic scatter to create a variable neutron beam from a monoenergetic neutron source. Radiat. Phys. Chem.112, 22-28. doi:10.1016/j.radphyschem.2015.03.002

\section{Footnotes}

1. computer code Monte Carlo N-Particle Transport version 5 (Los Alamos National Laboratory, Los Alamos, NM 87545, 2006). 


\section{Figure captions}

Fig. 1. A cutaway side view of the detector shield. This represents the layered steel and polyethylene design.

Fig. 2. A top-down view of the simulated room with the detector and source shield configuration with the water target in the middle.

Fig. 3. A graphical representation of the various shielding arrangements simulated in the realistic environment. Each layer is $0.05 \mathrm{~m}$ thick.

Fig. 4. The neutron flux for a variable energy neutron source at different locations around the a) layered polyethylene and steel and b) pure polyethylene detector shields.

Fig. 5. The detector neutron energy spectra for various aligned source and detector shield arrangements.

Fig. 6. The detector gamma ray energy spectra for various aligned source and detector shield arrangements.

Fig. 7. The detector neutron energy spectra for arrangements $7-12$, normalized by the spectra from a modified arrangement 5. There is a noticeable increase at $12.6 \mathrm{MeV}$, which is correlated to the elastic scatter of $14.1 \mathrm{MeV}$ neutrons off the nitrogen target at $\pi / 2$ radians.

Fig. 8. An example of how the modular shielding could be constructed so that there were no continuous seams between layers. The " $\mathrm{L}$ " shaped pieces form a rectilinear box can then be capped at one end to create either a source or detector shield.

Fig. 9. A way to design the various shield pieces so that they fit together more securely and limit the size and length of cracks at seams. 
Fig. 1

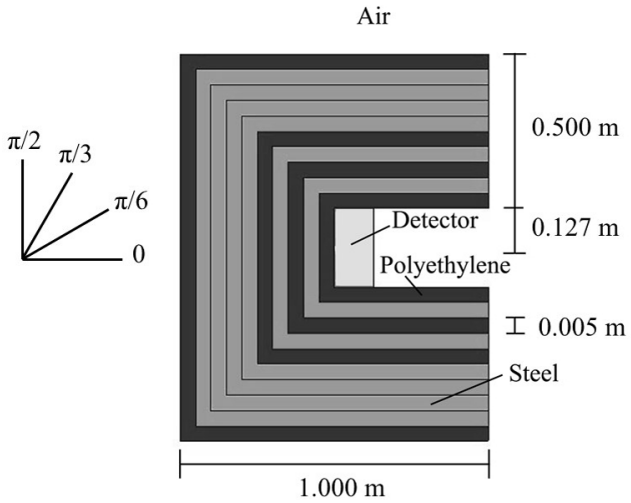


Fig. 2

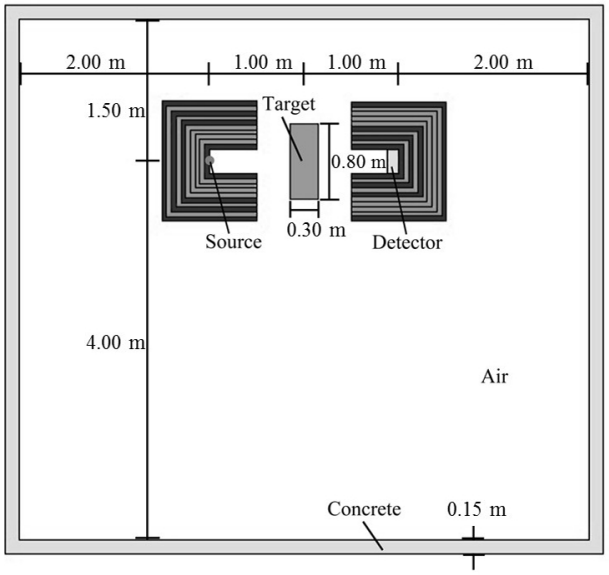


Fig. 3
$1 / 7$
$2 / 8$
$3 / 9$
$4 / 10$
$5 / 11$
$6 / 12$
Air

Source Detector Source Detector Source Detector Source Detector Source Detector Source Detector Source Detector
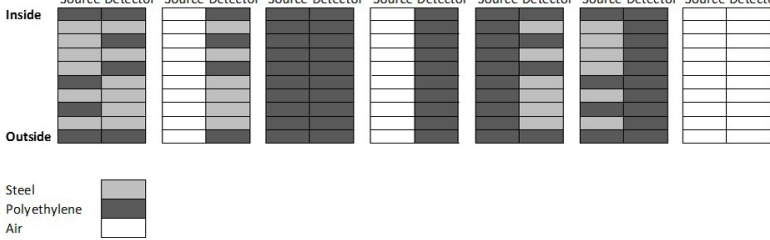
Fig. $4 a$

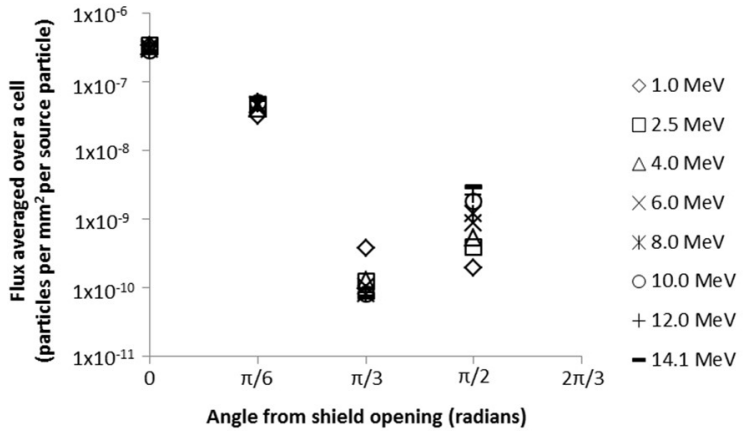


Fig. 4b

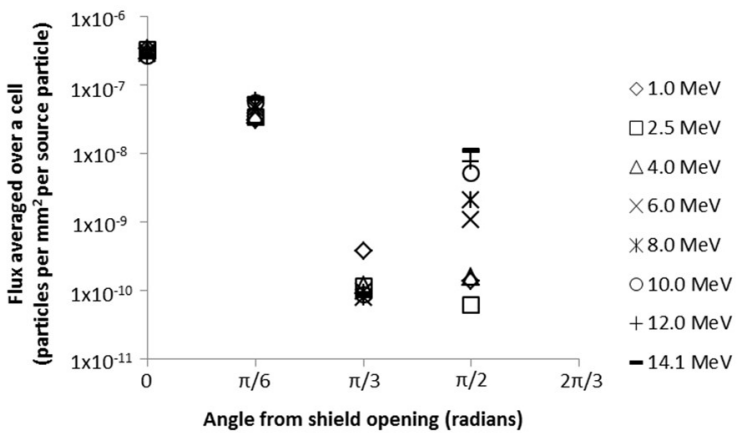


Fig. 5

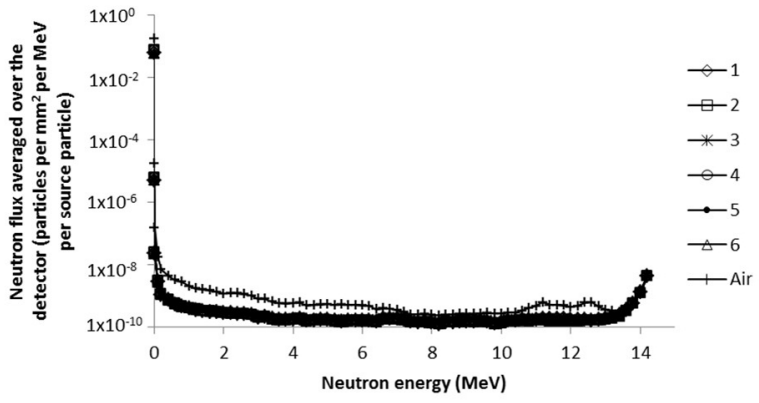


Fig. 6

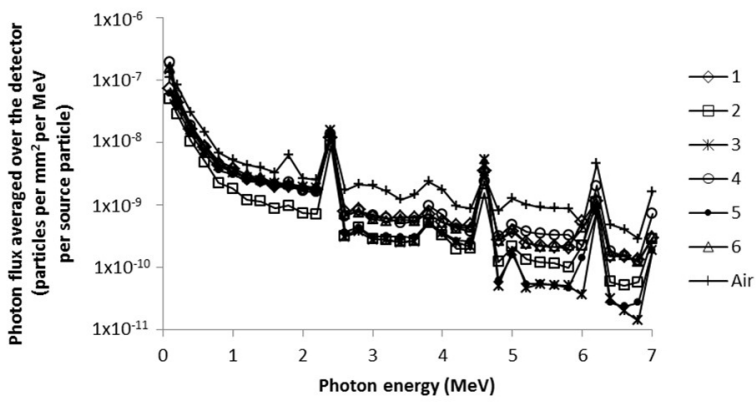


Fig. 7
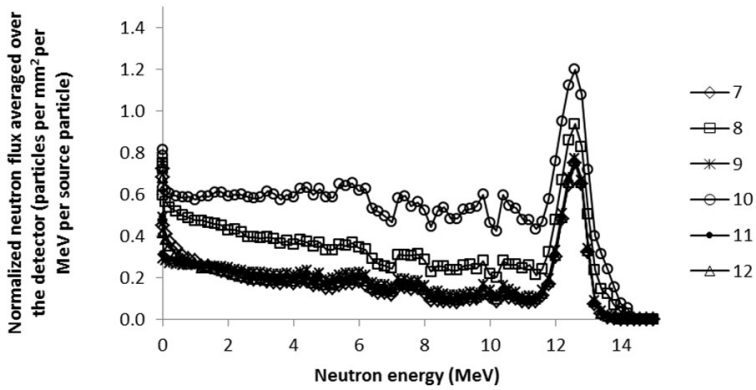

$\longleftarrow 12$ 
Fig. 8

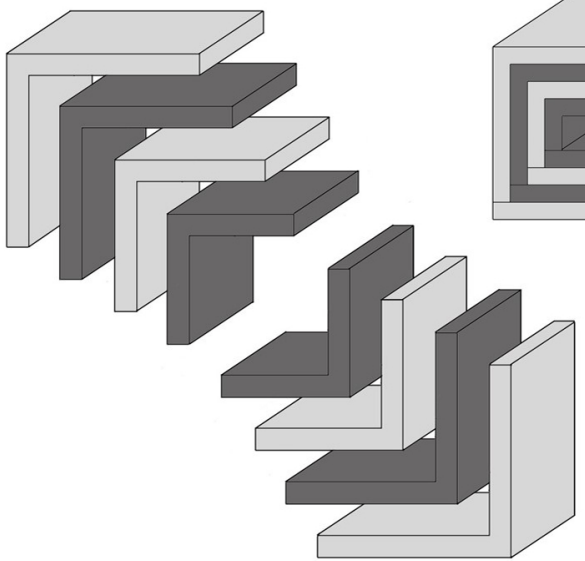


Fig. 9

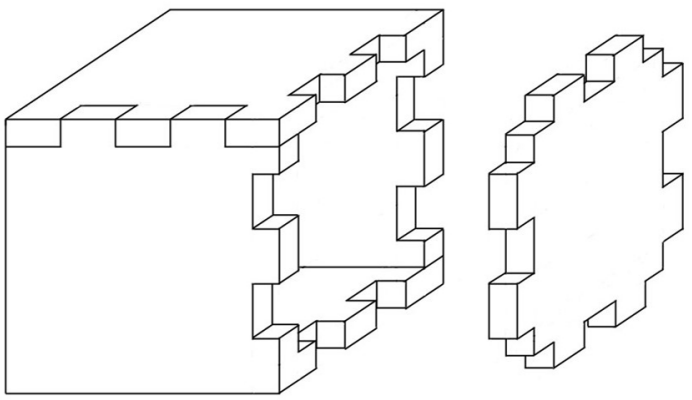

